\title{
MIXED-INTERPOLATED ELEMENTS FOR REISSNER-MINDLIN PLATES
}

\author{
FRANCO BREZZI \\ Istituto di Analisi Numerica del Consiglio Nazionale delle Ricerche, 27100 Pavia, Italy \\ KLAUS-JÜRGEN BATHE \\ Department of Mechanical Engineering, Massachusetts Institute of Technology, Cambridge, MA 02139, U.S.A. \\ MICHEL FORTIN \\ Département de Mathématique, Université Laval, Québec, Canada
}

\section{SUMMARY}

We present in this paper a procedure to establish Reissner-Mindlin plate bending elements. The procedure is based on the idea to combine known results on the approximation of Stokes problems with known results on the approximation of elliptic problems. The proposed elements satisfy the mathematical conditions of stability and convergence, and some of them promise to provide efficient elements for practical solutions.

\section{INTRODUCTION}

In this paper we present a family of finite element approximations of Reissner-Mindlin plates. The elements in the family are based on a mixed interpolation and include some already known clements, namely, the MITC4 $4^{1,2 *}$ and the MITC9 elements. ${ }^{3}$ We introduce many possible other elements and some of the new elements, as we shall see, have interesting and valuable features and might be useful in practical applications. Essentially, the whole family is based on a common idea, which is to combine in a proper way some known results on the approximation of Stokes problems with other known results on the approximation of linear elliptic problems. Such a combination, as we shall see, can be summarized in a list of five properties P1 to P5 that we may require for a finite element approximation of Reissner-Mindlin plate problems. Examples of approximations that satisfy our five properties are not difficult to find, and we detail some of them.

An outline of the paper is as follows.

In Section 2 we consider a sequence of plate bending problems $\left\{P_{t}\right\}$ with thickness $t$ going to zero. The sequence has the property that the corresponding solutions stay bounded (and do not vanish) when $t$ goes to zero. In the subsequent sections we use the sequence to study the shear locking phenomenon for various discretizations $\left\{P_{t h}\right\}$ of the problems of the sequence. We should, in principle, show that if an approximation satisfies our properties P1 to P5 then the solution of $P_{t h}$ converges to the solution of $P_{t}$ (as $h$ tends to zero) uniformly in $t$. This was done for instance in the

*The abbreviation MITC4 is used to designate our ciement based on 'mixed interpolated tensorial components with four nodes' and we use similar abbreviations for the other elements we introduce 
special case of the MITC4 element in Reference 4. However, it is difficult to perform such analysis in the general case and the technical details (as displayed in Reference 4) would be rather complicated. We choose here to perform a simplified analysis, as we did in Reference 3. Namely, we consider just the two limit problems $P_{0}$ and $P_{0 n}$ and we analyse convergence and error estimates only in this case. It is clear that, if $P_{i h}$ displays a 'good behaviour uniformly in $t$ ', then $P_{0 h}$ must also behave properly. Since the converse is not true, our analysis is not complete. However, we conjecture that the good behaviour for $t=0$ is a very reasonable test that can be of great help in designing a new element.

In Section 3 we introduce two examples of discretizations of $P_{t h}$, one based on the MITC7 element, a triangular element, and one based on the MITC9 element, a quadrilateral element.

In Section 4 we introduce the abstract properties P1 to P5 and we show that both the MITC7 element and the MITC9 element easily satisfy them.

In Section 5 we show, in an abstract framework, that if a finite element approximation $P_{\text {th }}$ satisfies P1 to P5 then $P_{0 h}$ converges to $P_{0}$ as $h$ tends to 0 , and we estimate the error for the rotations and transverse displacements. The abstract results are then applied to the two examples of the previous sections.

Finally, in Section 6, we show how P1 to P5 can be used as guidelines for designing new elements that have good stability and convergence properties (at least for the limit case $t=0$ ). Some other examples of finite element discretizations that satisfy P1 to P5 are built to illustrate the procedure.

Throughout the paper we shall use, for a vector $v=\left(v_{1}, v_{2}\right)$ the notation $v^{\perp}=\left(v_{2},-v_{1}\right)$. Different constants which are independent of $h$ might be indicated by the same letter $c$.

\section{THE SEQUENCE OF PROBLEMS AND THE LIMIT PROBLEM}

We consider the spaces $\Theta=\left(H_{0}^{1}(\Omega)\right)^{2}$ and $W=H_{0}^{1}(\Omega)$ and a load function $f$ given in $L^{2}(\Omega)$. The sequence of problems under consideration is ${ }^{5}$

$$
P_{t}: \operatorname{Inf}_{\theta \in \Theta, w \in W} \frac{t^{3}}{2} a(\theta, \theta)+\frac{\lambda t}{2}\|\theta-\nabla w\|_{0}^{2}-t^{3}(f, w)
$$

where $\frac{1}{2} t^{3} a(\theta, \theta)$ is the bending energy, $\frac{1}{2} \lambda t\|\theta-\nabla w\|_{0}^{2}$ is the shear energy and \|\|$_{0}$ and $($, represent respectively the norm and the inner product in $L^{2}(\Omega)$.

Assume now that we are given finite element subspaces $\Theta_{h} \subset \Theta$ and $W_{h} \subset W$. The corresponding discretized problem is described by

$$
\tilde{P}_{t h}: \operatorname{Inf}_{\theta_{h} \in \Theta_{h}, w_{h} \in w_{h}} \frac{t^{3}}{2} a\left(\theta_{h}, \theta_{h}\right)+\frac{\lambda t}{2}\left\|\theta_{h}-\nabla w_{h}\right\|_{0}^{2}-t^{3}\left(f, w_{h}\right)
$$

In general, $\tilde{P}_{t h}$ 'locks' for small $t .^{5}$ A common procedure is to reduce somehow the influence of the shear energy. We consider here the case in which the reduction is carried out in the following way: we assume that we are given a third finite element space, $\Gamma_{h}$, and a linear operator $R$ which takes values in $\Gamma_{h}$. Then we use $\left\|R\left(\theta_{h}-\nabla w_{h}\right)\right\|_{0}^{2}$ instead of $\left\|\theta_{h}-\nabla w_{h}\right\|_{0}^{2}$ in the shear energy. For the sake of simplicity we shall assume that

$$
R \nabla w_{h}=\nabla w_{h} \text { for all } w_{h} \in W_{h}
$$

We shall also assume that $R$ is a bounded operator, in the sense that there exist a constant $c$, independent of $h$, such that

$$
\|R \eta\|_{1} \leqslant c\left\|_{\eta}\right\|_{1} \quad \forall \eta \in \Theta_{h}
$$


The discretized problem then takes the final form

Setting

$$
P_{t h}: \operatorname{Inf}_{\theta_{h} \in \Theta_{h}, w_{h} \in W_{h}} \frac{t^{3}}{2} a\left(\theta_{h}, \theta_{h}\right)+\frac{\lambda t}{2}\left\|R \theta_{h}-\nabla w_{h}\right\|_{0}^{2}-t^{3}\left(f, w_{h}\right)
$$

$$
\gamma=\lambda t^{-2}(\theta-\nabla w) \text { and } \gamma_{h}=\lambda t^{-2}\left(R \theta_{h}-\nabla w_{h}\right)
$$

the Euler equations of $P_{t}$ and $P_{t h}$ are respectively

and

$$
\left.\begin{array}{l}
a(\theta, \eta)+(\gamma, \eta-\nabla \zeta)=(f, \zeta) \quad \forall \eta \in \Theta, \forall \zeta \in W \\
\gamma=\lambda t^{-2}(\theta-\nabla w)
\end{array}\right\}
$$

$$
\left.\begin{array}{l}
a\left(\theta_{h}, \eta\right)+\left(\gamma_{h}, R \eta-\nabla \zeta\right)=(f, \zeta) \quad \forall \eta \in \Theta_{h}, \forall \zeta \in W_{h} \\
\gamma_{h}=\lambda t^{-2}\left(R \theta_{h}-\nabla w_{h}\right)
\end{array}\right\}
$$

From now on we will limit ourselves to the analysis of the limit problems

$$
\left.\begin{array}{l}
a(\theta, \eta)+(\gamma, \eta-\nabla \zeta)=(f, \zeta) \quad \forall \eta \in \Theta, \quad \forall \zeta \in W \\
\theta=\nabla w
\end{array}\right\}
$$

and

$$
\left.\begin{array}{l}
a\left(\theta_{h}, \eta\right)+\left(\gamma_{h}, R \eta-\nabla \zeta\right)=(f, \zeta) \quad \forall \eta \in \Theta_{h}, \forall \zeta \in W_{h} \\
R \theta_{h}=\nabla w_{h}
\end{array}\right\}
$$

Remark. It is not difficult to show that (6) and (7) are the limit problems of (4) and (5) respectively, see for instance Reference 4 . In particular, the limit $w$ will be the solution corresponding to the Kirchhoff model. Note also that the limit $\gamma_{h}$ that appears in (7) will still belong to $R\left(\theta_{h}\right)-\nabla\left(W_{h}\right)$. Although we are not studying here the convergence of $\gamma_{h}$ to $\gamma$, the results given in Reference 6 with the discussion below give some insight into the behaviour of $\gamma_{h}$.

\section{THE MITC7 AND MITC9 ELEMENTS}

Following the discussion of the previous section, the finite element discretization is characterized by the choice of the finite element spaces $\Theta_{h}, W_{h}, \Gamma_{h}$ and by the choice of the linear operator $R$. It is obvious that these four choices are not independent. In particular, we need that

$$
R\left(\Theta_{h}\right) \subseteq \Gamma_{h}
$$

and we already assumed in (1) that

$$
(R-I)\left(\nabla W_{h}\right)=\{0\}
$$

where $I$ is the identity operator.

We now present two possible choices for $\Theta_{h}, W_{h}, \Gamma_{h}$ and $R$.

\section{The MITC7 element ${ }^{7}$}

We assume that we are given a regular sequence $\left\{T_{h}\right\}$ of decompositions of $\Omega$ into triangles $T$ (Reference 8 ) (for the sake of simplicity we assume that $\Omega$ is a polygon). For each triangle $T$ we set

$$
S_{7}(T)=\left\{\varphi\left|\varphi \in P_{3}, \varphi\right|_{e} \in P_{2} \text { on each edge } e \text { of } T\right\}
$$


Clearly $S_{7}$ is a finite dimensional linear space of dimension 7 . It can also be characterized as $S_{7}=P_{2} \oplus\left\{\lambda_{1} \lambda_{2} \lambda_{3}\right\}$ where $\lambda_{1} \lambda_{2} \lambda_{3}$ is the cubic bubble in $T$. As degrees of freedom in $S_{7}(T)$ we can clearly choose the values at the vertices, at the midpoint of the edges and at the barycentre of $T$.

We also set, in each triangle $T$,

$$
\begin{aligned}
R T_{1}(T)=\left\{\delta \mid \delta_{1}\right. & =a_{1}+b_{1} x+c_{1} y+y(d x+e y) \\
\delta_{2} & \left.=a_{2}+b_{2} x+c_{2} y-x(d x+e y)\right\}
\end{aligned}
$$

The space $R T_{1}(T)$ is a kind of "rotated Raviart-Thomas's space of order one.

We are now able to define our spaces. We set

$$
\begin{gathered}
\Theta_{h}=\left\{\eta\left|\eta \in\left(H_{0}^{1}\right)^{2}, \eta\right|_{T} \in\left(S_{7}(T)^{2} \forall T\right\}\right. \\
W_{h}=\left\{\zeta\left|\zeta \in H_{0}^{1}, \zeta\right|_{T} \in P_{2} \forall T\right\} \\
\Gamma_{h}=\left\{\delta|\delta|_{T} \in R T_{1}(T), \delta \cdot \tau\right. \text { continuous at the } \\
\text { interelement boundaries, } \delta \cdot \tau=0 \text { on } \partial \Omega\}
\end{gathered}
$$

where $\tau$ is the tangential unit vector to each edge of each element.

We have finally to introduce the reduction operator $R$. Its action on the current element is given as follows: for $\eta$ smooth in $T, R \eta$ in $T$ is the unique element in $R T_{1}(T)$ that satisfies

$$
\begin{array}{cc}
\int_{e}(\eta-R \eta) \cdot \tau p_{1}(s) \mathrm{d} s=0 & \forall e \text { edge of } T \\
\int_{T}(\eta-R \eta) \mathrm{d} x \mathrm{~d} y=0 & \forall p_{1}(s) \in P_{1}(e)
\end{array}
$$

It is easy to see that (14) and (15) characterize $R \eta$ in $T$ in a unique way. ${ }^{9}$ It is also clear that, if $\eta$ is continuous in $\Omega$, then the $R \eta$ constructed element by element through (14), (15) actually belongs to $\Gamma_{h}$ (because (14) ensures the continuity of $(R \eta) \cdot \tau$ at the interelement boundaries).

The properties of the MITC7 element will be discussed in the next section.

\section{The MITC9 element ${ }^{3}$}

Assume, for the sake of simplicity, that $\Omega$ is now a rectangle divided into rectangles $K$. We set

$$
\begin{aligned}
& \Theta_{h}=\left\{\eta\left|\eta \in\left(H_{0}^{1}(\Omega)\right)^{2}, \eta\right|_{K} \in\left(Q_{2}\right)^{2} \forall K\right\} \\
& W_{h}=\left\{\zeta\left|\zeta \in H_{0}^{1}(\Omega), \zeta\right|_{K} \in Q_{2}^{r} \forall K\right\}
\end{aligned}
$$

where $Q_{2}$ is the space of polynomials of degree $\leqslant 2$ in each variable (corresponding to a 9 node element) and $Q_{2}^{r}$ is its usual serendipity reduction (corresponding to an 8 node element). In order to introduce the space $\Gamma_{h}$ we define first the space of polynomials

$$
\begin{aligned}
G=\left\{\delta \mid \delta_{1}\right. & =a_{1}+b_{1} x+c_{1} y+d_{1} x y+e_{1} y^{2} \\
\delta_{2} & \left.=a_{2}+b_{2} x+c_{2} y+d_{2} x y+e_{2} x^{2}\right\}
\end{aligned}
$$

which is some kind of rotated Brezzi-Douglas Fortin-Marini space. ${ }^{10}$

We introduce now the space $\Gamma_{h}$ :

$$
\Gamma_{h}=\left\{\delta \mid \delta_{K} \in G \forall K, \delta \cdot \tau \text { continuous at the interelement boundaries, } \delta \cdot \tau=0 \text { on } \partial \Omega\right\}
$$


Further, we define the action of the reduction operator $R$ on the current element $K$ in the following way: for $\eta$ smooth in $K,\left.R \eta\right|_{K}$ is the unique element in $G$ that satisfies

$$
\begin{array}{cl}
\int_{e}(\eta-R \eta) \cdot \tau p_{1}(s) \mathrm{d} s=0 & \forall e \text { an edge of } K \\
& \forall p_{1}(s) \text { polynomial of degree } \leqslant 1 \text { on } e
\end{array}
$$

Here again (1) is satisfied.

Remark. Note that both (14) and (20) can be satisfied (for $\eta \in \Theta_{h}$ ) by tying the values $\eta \cdot \tau=(R \eta) \cdot \tau$ at the two Gauss points of each edge. On the contrary (15) and (21) cannot be satisfied by simple one-point tying. If we change these conditions into the condition " $\eta=R \eta$ at the centre of the element' our analysis is not applicable anymore, although numerical experiments may show good element behaviour even in this case. ${ }^{11}$

It is also clear that for non-affine elements $R$ should be defined by covariant interpolations (see References 1,2 and 11).

\section{PROPERTIES P1 TO P5}

We shall now introduce our five properties P1 to P5 and check that they are satisfied with the choices of the previous section.

The first property was already introduced in (1), which is equivalent to asking that

$$
\nabla W_{h} \subseteq \Gamma_{h}
$$

It is very easy to check, in each application, whether $\mathrm{P} 1$ is satisfied or not. First of all, let us note that if $W_{h}$ is a piecewise polynomial subspace of $H_{0}^{1}(\Omega)$ (and hence its members are continuous at the interfaces and vanish on $\partial \Omega$ ) then for any $\zeta \in W_{h}$ we have that $(\nabla \zeta) \cdot \tau$ is continuous at the interfaces and vanishes at $\partial \Omega$. Hence for the MITC7 element we have only to check that $\left.\nabla \zeta\right|_{T} \in R T_{1}(T)$ (for all $T$ ) whenever $\zeta \in P_{2}$ in $T$ (which is obvious). Similarly, for the MITC9 we have only to check that $\left.\nabla \zeta\right|_{K} \in G$ (for all $K$ ) whenever $\zeta \in Q_{2}^{r}$ in $T$ (which is also obvious). We also point out that, as we have seen, to check if $\mathrm{P} 1$ holds or not in a particular case is very easy but, nevertheless, $P 1$ is a very strong assumption that can severely limit our choice of available elements.

The next properties introduce a new space that we call $Q_{h}$. The space $Q_{h}$ is never used in the computations but its existence (with the suitable properties given below) is crucial for our analysis. We anticipate that for both elements, i.e. the MITC7 and MITC9 elements, the space $Q_{h}$ will be

$$
Q_{h}=\text { \{ piecewise linear, a priori discontinuous functions }
$$

We are now able to introduce our second property,

$$
\operatorname{rot} \Gamma_{h} \subseteq Q_{h}
$$

We recall that in $\mathbb{R}^{2}$ for a vector $\delta$ and a scalar $\zeta$ we have, by definition,

$$
\begin{aligned}
& \operatorname{rot} \delta:=\frac{\partial \delta_{2}}{\partial x}-\frac{\partial \delta_{1}}{\partial y}=\operatorname{div} \delta^{\perp} \\
& \operatorname{rot} \zeta:=\left(\frac{\partial \zeta}{\partial y},-\frac{\partial \zeta}{\partial x}\right)=(\nabla \zeta)^{\perp}
\end{aligned}
$$


To check that P2 is satisfied by the MITC7 and MITC9 elements with the choice (22) is elementary. We point out, however, that the continuity of $\delta \cdot \tau$ at the interfaces ensures that rot $\delta$ does not have Dirac delta measures at the interfaces.

Whether $\mathbf{P} 2$ is restrictive or not can be appreciated only after stating the next property.

'The pair $\Theta_{h}, Q_{h}$ is good for the Stokes problem'

Property P3 clearly needs some explanation. What we really need is that for any $\theta$ smooth enough in $\left(H_{0}^{1}(\Omega)\right)^{2}$ with rot $\theta=0$ there exists $\theta^{\mathrm{l}}$ in $\Theta_{h}$ such that

$$
\begin{array}{ll}
\left\|\theta-\theta^{\mathbf{I}}\right\|=O\left(h^{r}\right) & \text { for some } r>0 \\
\int_{\Omega} \operatorname{rot} \theta^{\prime} q_{h} \mathrm{~d} x \mathrm{~d} y=0 & \text { for all } q_{h} \in Q_{h}
\end{array}
$$

Obviously, the larger is $r$, the better our Reissner-Mindlin element will be.

In order to identify the relationships between $P 3$ and (25), (26) we can argue in the following way. Let us assume that we are given a smooth $\theta \in\left(H_{0}^{1}(\Omega)\right)^{2}$ with $\operatorname{rot} \theta=0$ in $\Omega$. Set $\chi:=-\Delta \theta$ and consider the problem

$$
\left.\begin{array}{l}
\text { find }(\tilde{\theta}, p) \in\left(H_{0}^{1}(\Omega)\right)^{2} \times L^{2} / \mathbb{R} \text { such that } \\
\left.\begin{array}{l}
-\Delta \tilde{\theta}+\operatorname{rot} p=\chi \\
\operatorname{rot} \tilde{\theta}=0
\end{array}\right\} \text { in } \Omega
\end{array}\right\}
$$

It is obvious that (27) has a unique solution $\tilde{\theta}=\theta, p=0$. On the other hand the numerical approximation of (27) is clearly as difficult (or as easy) as for a Stokes problem. Let us consider the following discretization of (27):

Find $\left(\theta^{\mathrm{I}}, p_{h}\right) \in \Theta_{h} \times Q_{h} / \mathbb{R}$ such that

$$
\left.\begin{array}{l}
\int_{\Omega} \operatorname{grad} \theta^{\prime}: \operatorname{grad} \eta \mathrm{d} x \mathrm{~d} y-\int_{\Omega} p_{h} \operatorname{rot} \eta \mathrm{d} x \mathrm{~d} y=\int_{\Omega} \chi \cdot \eta \mathrm{d} x \mathrm{~d} y \quad \forall \eta \in \Theta_{h} \\
\int_{\Omega} q_{h} \operatorname{rot} \theta^{\mathrm{I}} \mathrm{d} x \mathrm{~d} y=0 \quad \forall q_{h} \in Q_{h}
\end{array}\right\}
$$

It is clear that if $\left(\Theta_{h}, Q_{h}\right)$ make (28) a stable and convergent approximation for problem (27) (which is surely true if the pair $\left(\Theta_{h}, Q_{h}\right)$ is a good pair (velocities, pressure) for approximating Stokes problems) then the solution $0^{\mathrm{I}}$ will satisfy (25), (26).

Let us see what happens for the MITC7 and MITC9 elements. For the MITC7 element we refer to the Crouzeix-Raviart element, whose stability and convergence properties for Stokes problems (and hence for (27)) are well known (see Reference 12). For the MITC9 element we refer to the $Q_{2}-P_{1}$ element which is also known to be very good. ${ }^{6,13,14}$ Hence in both cases P3 is satisfied. Moreover, we have $r=2$ in (25).

Remark. It is clear that a sufficient condition in order to satisfy P3 is the inf-sup condition:

$$
\inf _{q_{h} \in Q_{h}} \sup _{\eta_{h} \in \Theta_{h}} \frac{\int q_{h} \operatorname{rot} \eta_{h} \mathrm{~d} x \mathrm{~d} y}{\left\|\eta_{h}\right\|_{H^{1}}\left\|q_{h}\right\|_{L^{2} / \mathbb{R}}} \geqslant c>0
$$

with $c$ independent of $h$.

The relationship between Stokes problems and the Reissner-Mindlin problem is actually deeper than it may appear here. The interested reader can find much more detail on this subject in 
Reference 15, where the Reissner-Mindlin problem is actually represented as two Poisson problems and one Stokes problem.

The fourth property could be stated as

The pair $\Gamma_{h}, Q_{h}$ is good for elliptic problems

However, we want to state this property in a more precise way. For this purpose we define the $L^{2}$. projection operator $P_{h}: L^{2} \rightarrow Q_{h}$ as

$$
\left(P_{h} q, q_{h}\right)=\left(q, q_{h}\right) \quad \forall q \in L^{2}, \forall q_{h} \in Q_{h}
$$

Then our fourth property is

$$
\text { For all } \eta \in\left(H^{1}(\Omega)\right)^{2} \quad \text { we have } \operatorname{rot} R \eta=P_{h} \operatorname{rot} \eta
$$

Note that, since $R \eta \in \Gamma_{h}$, rot $R \eta$ will automatically be in $Q_{h}$ because of property (P2). The question is whether rot $R \eta$ is equal to $P_{h}$ rot $\eta$ or not.

Let us check whether this property is fulfilled for the MITC7 element. Using (30) we have to prove that

$$
\int_{\Omega}(\operatorname{rot} R \eta) q_{h} \mathrm{~d} x \mathrm{~d} y=\int_{\Omega}(\operatorname{rot} \eta) q_{h} \mathrm{~d} x \mathrm{~d} y \quad \forall q_{h} \in Q_{h}
$$

Let us consider one triangle $T$. We have

$$
\begin{aligned}
\int_{T}(\operatorname{rot} R \eta) q_{h} \mathrm{~d} x \mathrm{~d} y & =-\int_{T} R \eta \cdot \operatorname{rot} q_{h} \mathrm{~d} x \mathrm{~d} y+\int_{\partial T}(R \eta) \cdot \tau q_{h} \mathrm{~d} s=(\text { using (15), (22)) } \\
& =-\int_{T} \eta \cdot \operatorname{rot} q_{h} \mathrm{~d} x \mathrm{~d} y+\int_{\partial T}(R \eta) \cdot \tau q_{h} \mathrm{~d} s=\text { (using (14), (22)) } \\
& =-\int_{T} \eta \cdot \operatorname{rot} q_{h} \mathrm{~d} x \mathrm{~d} y+\int_{\partial T} \eta \cdot \tau q_{h} \mathrm{~d} s=\int_{T}(\operatorname{rot} \eta) q_{h} \mathrm{~d} x \mathrm{~d} y
\end{aligned}
$$

Clearly, (32) implies (31), which is equivalent to P4.

The proof that the MITC9 element also satisfies P4 is formally identical to (32). We have just to use (18), (19) instead of (14), (15) respectively.

Remark. The property P4 is called the commuting diagram property (see Reference 16) in the context of mixed methods for second order elliptic problems.

The last property is also restrictive, in particular when joined with $\mathrm{P} 1$.

$$
\left.\begin{array}{l}
\text { If } \delta_{h} \in \Gamma_{h} \text { is such that } \operatorname{rot} \delta_{h}=0 \\
\text { then } \delta_{h} \in \nabla W_{h}
\end{array}\right\}
$$

Note that, in $\mathbb{R}^{2}$, the condition

$$
\operatorname{rot} \delta_{h}=0
$$

already implies that

$$
\delta_{h}=\nabla \zeta \quad \text { for some } \zeta \in H_{0}^{1}
$$

In particular the condition $\zeta=0$ on $\partial \Omega$ comes from the property ' $\delta_{h} \cdot \tau=0$ on $\partial \Omega$ ' in the definition of $\Gamma_{h}$.

What we have to check here is that such a $\zeta$ actually belongs to $W_{h}$. 
Let us consider the MITC7 element first. If $\delta_{h} \in \Gamma_{h}$ then, in each triangle, $\delta_{h} \in R T_{1}$ (see (13)) and therefore $\delta_{h}$ has the form (10). It is easy to check that the condition rot $\delta_{h}=0$ implies, in particular, $d=e=0$ in (10). Hence $\delta_{h}$ is actually a polynomial of degree $\leqslant 1$ in each $T$. Therefore, the function $\zeta$ in (34) must be a polynomial of degree $\leqslant 2$ in each $T$ and hence it belongs to $W_{h}$ (see (12)).

Let us now check that the MITC9 element also satisfies P5. This is easier. If $\delta_{h} \in \Gamma_{h}$ it will have the form (18) in each $K$. It is easy to check that this implies that $\zeta$ in (34) is a polynomial in $Q_{2}$ that does not contain the term $x^{2} y^{2}$. Hence $\left.\zeta\right|_{K} \in Q_{2}^{r}$ and $\zeta \in W_{h}$ (see (17)).

\section{ABSTRACT ERROR ANALYSIS ASSUMING P1 TO P5}

Let us go back to problems (6) and (7) which we restate here for the convenience of the reader:

and

$$
\left.\begin{array}{l}
a(\theta, \eta)+(\gamma, \eta-\nabla \zeta)=(f, \zeta) \quad \forall \eta \in \Theta, \forall \zeta \in W \\
\theta=\nabla w
\end{array}\right\}
$$

$$
\left.\begin{array}{l}
a\left(0_{h}, \eta\right)+\left(\gamma_{h}, R \eta-\nabla \zeta\right)=(f, \zeta) \quad \forall \eta \in \Theta_{h}, \quad \forall \zeta \in W_{h} \\
R \theta_{h}=\nabla w_{h}
\end{array}\right\}
$$

We have the following abstract result.

\section{Theorem 1}

Let $(\theta, w)$ and $\left(\theta_{h}, w_{h}\right)$ be the solutions of $(35),(36)$ respectively. Assume that there exists a space $Q_{h}$ such that the system $\left(\Theta_{h}, W_{h}, \Gamma_{h}, R, Q_{h}\right)$ satisfies PI to P5. Then we have the following error bounds:

$$
\begin{aligned}
& \left\|\theta-\theta_{h}\right\|_{1} \leqslant c\left[\sup _{\eta \in \Theta_{h}} \frac{(\gamma, \eta-R \eta)}{\|\eta\|_{1}}+\left\|\theta-\theta^{\mathrm{I}}\right\|_{1}\right] \\
& \left\|\nabla w-\nabla w_{h}\right\|_{0} \leqslant c\left[\|\theta-R \theta\|_{0}+\left\|\theta-\theta_{h}\right\|_{1}\right]
\end{aligned}
$$

where $c$ is independent of $h$ and $0^{\mathrm{I}} \in \Theta_{h}$ is given in $P 3$.

Proof. We recall first that there exists a positive constant $\alpha$ such that

$$
a(\eta, \eta) \geqslant \alpha\|\eta\|_{1}^{2} \quad \forall \eta \in \Theta
$$

Then we consider P3 and we have the existence of an element $\theta^{\mathrm{I}}$ in $\Theta_{h}$ that satisfies (26). If we write (26) in the form

$$
P_{h} \operatorname{rot} \theta^{\mathrm{I}}=0
$$

(which is allowed by (30)) we can now use P4 and obtain

$$
\operatorname{rot} R \theta^{\mathrm{I}}=0
$$

Since $R \theta^{\mathrm{I}}$ clearly belongs to $\Gamma_{h}$, we can use P5 and have that there exists a unique $w^{\mathrm{I}}$ in $W_{h}$ such that

$$
\nabla w^{1}=R \theta^{1}
$$

We are now able to prove (37). We set

$$
\varepsilon_{\theta}=\theta_{h}-\theta^{\mathrm{I}} \quad \varepsilon_{w}=w_{h}-w^{\mathrm{I}}
$$


and we note that, from (42) and (36),

We have

$$
R \varepsilon_{\theta}=\nabla \varepsilon_{w}
$$

$$
\begin{aligned}
\alpha\left\|\varepsilon_{\theta}\right\|_{1}^{2} \leqslant(\text { use }(39)) \leqslant a\left(\varepsilon_{\theta}, \varepsilon_{\theta}\right) & =(\text { use }(43)) \\
& =a\left(\theta_{h}-\theta^{\mathrm{I}}, \varepsilon_{\theta}\right)=(\text { add and subtract } \theta) \\
& =a\left(\theta_{h}-\theta, \varepsilon_{\theta}\right)+a\left(\theta-\theta^{\mathrm{I}}, \varepsilon_{\theta}\right)=\left(\text { use }(35),(36) \text { with } \eta=\varepsilon_{\theta}, \zeta=0\right) \\
& =-\left(\gamma_{h}, R \varepsilon_{\theta}\right)+\left(\gamma, \varepsilon_{\theta}\right)+a\left(\theta-\theta^{\mathrm{I}}, \varepsilon_{\theta}\right) \\
& =\left(\operatorname{add} \text { and subtract }\left(\gamma, R \varepsilon_{\theta}\right)\right) \\
& =\left(\gamma-\gamma_{h}, R \varepsilon_{\theta}\right)+\left(\gamma, \varepsilon_{\theta}-R \varepsilon_{\theta}\right)+a\left(\theta-\theta^{\mathrm{I}}, \varepsilon_{\theta}\right)=(\text { use (44)) } \\
& =\left(\gamma-\gamma_{h}, \nabla \varepsilon_{w}\right)+\left(\gamma, \varepsilon_{\theta}-R \varepsilon_{\theta}\right)+a\left(\theta-\theta^{\mathrm{I}}, \varepsilon_{\theta}\right) \\
& =\left(\text { use }(35),(36) \text { in the first term with } \eta=0, \zeta=\varepsilon_{w}\right) \\
& =\left(\gamma, \varepsilon_{\theta}-R \varepsilon_{\theta}\right)+a\left(\theta-\theta^{\mathrm{l}}, \varepsilon_{\theta}\right)
\end{aligned}
$$

We use now the obvious facts that

and

$$
\left(\gamma, \varepsilon_{\theta}-R \varepsilon_{\theta}\right)=\frac{\left(\gamma, \varepsilon_{\theta}-R \varepsilon_{\theta}\right)}{\left\|\varepsilon_{\theta}\right\|_{1}}-\left\|\varepsilon_{\theta}\right\|_{1} \leqslant \sup _{\eta \in \Theta_{h}} \frac{(\gamma, \eta-R \eta)}{\|\eta\|_{1}}\left\|\varepsilon_{\theta}\right\|_{1}
$$

$$
a\left(\theta-\theta^{\mathbf{l}}, \varepsilon_{\theta}\right) \leqslant c\left\|\theta-\theta^{\mathbf{l}}\right\|_{1}\left\|\varepsilon_{\theta}\right\|_{1}
$$

From (45), (46) and (47) we have

$$
\alpha\left\|\varepsilon_{\theta}\right\|_{1}^{2} \leqslant\left\{\sup _{\eta \in \Theta_{h}} \frac{(\gamma, \eta-R \eta)}{\|\eta\|_{1}}+c\left\|\theta-\theta^{\mathrm{I}}\right\|_{1}\right\}\left\|\varepsilon_{\theta}\right\|_{1}
$$

which implies

$$
\left\|\varepsilon_{\theta}\right\|_{1} \leqslant c\left\{\sup _{\eta \in \Theta_{h}}\left\{(\gamma, \eta-R \eta) /\|\eta\|_{1}\right\}+\left\|\theta-\theta^{1}\right\|_{1}\right\}
$$

Then we obtain (37) from the definition of $\varepsilon_{\theta}$ in (43), from (49) and from the triangle inequality $\left\|\theta-\theta_{h}\right\|_{1} \leqslant\left\|\theta-\theta^{\mathrm{I}}\right\|_{1}+\left\|\theta^{\mathrm{I}}-\theta_{h}\right\|_{1}$. Let us now prove (38). Since $\nabla w_{h}=R \theta_{h}$ (see (36)) we have

$$
\left\|\nabla w-\nabla w_{h}\right\|_{0}=\left\|\theta-R \theta_{h}\right\|_{0} \leqslant\|\theta-R \theta\|_{0}+\left\|R \theta-R \theta_{h}\right\|_{0}
$$

From (50) and (2) we have (38).

Remark. Theorem 1 expresses the crrors $\left\|\theta-\theta_{h}\right\|_{1}$ and $\left\|\nabla w-\nabla w_{h}\right\|_{0}$ in terms of the error in the solution of Stokes problem' (25) (for $\left\|\theta-\theta^{1}\right\|_{1}$ ) and of the approximation properties of the operator $R$. It is clear that this last component in the error appears because we make an error when we introduce the shear interpolations using $R$ instead of the identity (see the definition of $P_{t h}$ from $\widetilde{P}_{\text {th }}$ in Section 2). On the other hand, in the theory of mixed methods for second-order elliptic problems, if the commuting diagram property $\mathrm{P} 4$ holds, the error bounds are basically obtained in terms of $\|R-I\|$. In a sense we would then say that the error in our element constructions (using $\mathrm{P} 1$ to $\mathrm{P} 5$ ) is the sum of the "error in the Stokes problem' plus the 'error for the mixed method in elliptic problems'. 
Let us see now what we obtain from (37) and (38) for our MITC7 and MITC9 elements. We have already seen in discussing P3 that in both cases we have $\left\|\theta-\theta^{\mathrm{I}}\right\|_{1}=O\left(h^{2}\right)$. More precisely

$$
\left\|\theta-\theta^{\mathrm{I}}\right\|_{1} \leqslant c h^{2}\|\theta\|_{3}
$$

It is also clear that in both cases we also have for $1 \leqslant s \leqslant 3$,

$$
\|\theta-R \theta\|_{0} \leqslant c h^{s}\|\theta\|_{s}
$$

so that we have to deal only with the (more delicate) term containing the sup expression. Let $\tilde{\gamma}$ be the projection of $\gamma$ onto the space of piecewise constants. Then using (15) (respectively (21)) we have

so that

$$
(\tilde{\gamma}, \eta-R \eta)=0 \quad \forall \eta \in \Theta
$$

Hence we have

$$
\begin{aligned}
(\gamma, \eta-R \eta) & =(\gamma-\tilde{\gamma}, \eta-R \eta) \\
& \leqslant\|\gamma-\tilde{\gamma}\|_{0}\|\eta-R \eta\|_{0} \leqslant(\text { using (52) with } s=1) \\
& \leqslant\|\gamma-\tilde{\gamma}\|_{0} c h\|\eta\|_{1}
\end{aligned}
$$

$$
\sup _{\eta \in \Theta}\left\{(\gamma, \eta-R \eta) /\|\eta\|_{1}\right\} \leqslant c h\|\gamma-\tilde{\gamma}\|_{0}
$$

From (55) and classical approximation theory we have finally (always for both cases)

$$
\sup _{\eta \in \Theta}\left\{(\gamma, \eta-R \eta) /\|\eta\|_{1}\right\} \leqslant c h^{2}\|\gamma\|_{1}
$$

Inserting (51), (52) and (56) into (37) and (38) we have now the error bounds for the MITC7 and MITC9 elements,

$$
\left\|\theta-\theta_{h}\right\|_{1}+\left\|\nabla w-\nabla w_{h}\right\|_{0} \leqslant \operatorname{ch}^{2}\left(\|\theta\|_{3}+\|\gamma\|_{1}\right)
$$

\section{HOW TO DESIGN A NEW ELEMENT}

We can summarize the results of the previous sections by giving indications on how we can design new Reissner-Mindlin plate bending elements. We shall split the procedure into three steps.

Step 1. We start from a pair of spaces $\left(\Theta_{h}, Q_{h}\right) \subset\left(H_{0}^{1}\right)^{2} \times L^{2}(\Omega)$ that satisfy the inf-sup condition (29). This, as we have seen, implies that P3 will be satisfied. Many of such pairs can be found in the literature for Stokes problems (see for instance the books 7 and 17). We consider here some examples.

Example 1. The $Q_{3}-P_{2}$ element for rectangles. This is, in some way, the extension of the MITC9 element to a higher degree. We take

$$
\Theta_{h}=\left\{\eta\left|\eta \in\left(H_{0}^{1}(\Omega)\right)^{2}, \eta\right|_{K} \in Q_{3} \forall K\right\}
$$

where $Q_{3}$ is the set of polynomials of degree $\leqslant 3$ in each variable; we also take

$$
Q_{h}=\left\{q\left|q \in L^{2}(\Omega), q\right|_{K} \in P_{2} \forall K\right\}
$$

It is easy to see that $\mathrm{P} 3$ is satisfied with $r=3$.

Example 2. The $P_{3} \oplus B_{4}-P_{2}$ element for triangles. This can be seen as an extension of the MITC7 element. We set at first

$$
S_{12}(T)=\left\{\varphi\left|\varphi \in P_{4}, \varphi\right|_{e} \in P_{3} \text { on edge } e \text { of } T\right\}
$$


and then

$$
\begin{aligned}
& \Theta_{h}=\left\{\eta\left|\eta \in\left(H_{0}^{1}(\Omega)\right)^{2}, \eta\right|_{T} \in\left(S_{12}(T)\right)^{2} \forall T\right\} \\
& Q_{h}=\left\{q\left|q \in L^{2}(\Omega), q\right|_{T} \in P_{2} \forall T\right\}
\end{aligned}
$$

It is also easy to see that $\mathrm{P} 3$ is satisfied with $r=3$.

Other examples can be inferred from the existing literature. For instance, for triangular elements, we can consider $P_{2}-P_{0}$ elements or $P_{k}-P_{k-1}$ for $k \geqslant 4$. The corresponding value of $r$ in (25) will be 1 and $k$, respectively.

Step 2. We consider now a pair $\left(\Gamma_{h}, Q_{h}\right) \subset H_{0}(\operatorname{rot} ; \Omega) \times L^{2}(\Omega)$ that satisfies the commuting diagram property. We recall that

$$
H_{0}(\operatorname{rot} ; \Omega)=\left\{\delta \mid \delta \in\left(L^{2}(\Omega)\right)^{2}, \operatorname{rot} \delta \in L^{2}(\Omega), \delta \cdot \tau=0 \text { on } \partial \Omega\right\}
$$

By setting

$$
\mathscr{H}=\left\{\delta \mid \delta \in\left(H^{1}(\Omega)\right)^{2}, \delta \cdot \tau=0 \text { on } \partial \Omega\right\}
$$

the commuting diagram property (CDP) reads:

There exists a uniformly bounded (see (2)) linear operator $R: \mathscr{H} \rightarrow \Gamma_{h}$ such that the following diagram commutes

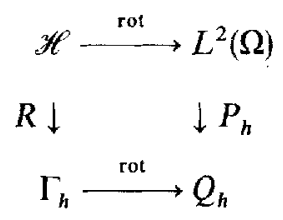

where $P_{h}$ is the $L^{2}$-projection on $Q_{h}(30)$.

Note that this will imply that P2 and P4 hold.

We explicitly point out that the space $Q_{h}$ in Step 2 must be the same as in Step 1. Pairs $\Gamma_{h}, Q_{h}$ satisfying the CDP are also easily found in the literature. Typical families are presented in References 9, 10, 18 and 19. See also Reference 7 for a general presentation. For our two new examples that we are building in this section, we have that $Q_{h}$ consists of piecewise quadratics. Hence, we look for spaces $\Gamma_{h}$ that work in conjunction with piecewise quadratics. For the triangular case, we have essentially three choices in the literature: We describe them on the current triangle $T$

$$
\begin{aligned}
& \text { (i) } \mathrm{BDM}_{3}=\left\{\delta \mid \delta \in\left(P_{3}\right)^{2}\right\} \\
& \text { (ii) } \mathrm{BDFM}_{3}=\left\{\delta\left|\delta \in\left(P_{3}\right)^{2}, \delta \cdot \tau\right|_{e} \in P_{2} \forall e\right\} \\
& \text { (iii) } \mathrm{RT}_{2}=\left\{\delta \mid \delta \in\left(P_{2}\right)^{2} \oplus \underline{x}^{1} P_{2}\right\} .
\end{aligned}
$$

where in (iii) (and in what follows) $\underline{x}^{\perp}$ is the vector $(y,-x)$.

We delay the choice until the next step.

For rectangular elements we have only two choices:

$$
\left.\begin{array}{l}
\text { (iv) } \mathrm{BDM}_{3}=\left\{\delta \mid \delta \in\left(P_{3}\right)^{2} \oplus \operatorname{grad}\left(x y^{4}\right) \oplus \operatorname{grad}\left(x^{4} y\right)\right\} \\
\text { (v) } \mathrm{BDFM}_{3}=\left\{\delta \mid \delta_{1} \in P_{3} \backslash x^{3}, \delta_{2} \in P_{3} \backslash y^{3}\right\}
\end{array}\right\}
$$

Again we delay the choice until the next step. Notice that if we use spaces $Q_{n}$ made of discontinuous polynomials of degree $k-1$, the menu of choices (i)-(v), here given for $k=3$ is fairly 
general as far as the present literature is concerned. Analogous spaces for $\Gamma_{h}$ can be constructed for other values of $k$.

Step 3. We have now to build the space $W_{h}$ in order to satisfy P1 and P5. Summarizing we need that

$$
\nabla W_{h}=\left\{\delta \mid \delta \in \Gamma_{h}, \operatorname{rot} \delta=0\right\}
$$

Accordingly we have after some simple computations for our examples (see (65)):

$$
\begin{aligned}
\text { (i) } W_{h} & =\left\{\zeta\left|\zeta \in H_{0}^{1}(\Omega), \zeta\right|_{T} \in P_{4} \forall T\right\} \\
\text { (ii) } W_{h} & =\left\{\zeta\left|\zeta \in H_{0}^{1}(\Omega), \zeta\right|_{T} \in S_{12} \forall T\right\} \\
\text { (iii) } W_{h} & =\left\{\zeta\left|\zeta \in H_{0}^{1}(\Omega), \zeta\right|_{T} \in P_{3} \forall T\right\} \\
\text { (iv) } W_{h} & =\left\{\zeta\left|\zeta \in H_{0}^{1}(\Omega), \zeta\right|_{K} \in P_{4} \oplus x^{4} y \oplus x y^{4}\right\} \\
\text { (v) } W_{h} & =\left\{\zeta\left|\zeta \in H_{0}^{1}(\Omega), \zeta\right|_{K} \in Q_{3} \cap P_{4} \forall K\right\}
\end{aligned}
$$

It is clear that we want to discard (iv) which requires degrees of freedom that are too different from the ones of $\Theta_{h}$. Hence (v) is the choice for the rectangular element. As far as the triangular element is concerned, (i) seems unnecessarily expensive, since we shall have $O\left(h^{3}\right)$ error bounds in any case. The choice between (ii) and (iii) is more delicate, since (iii) is a smaller space but on the other hand (ii) will use the same degrees of freedom that we use in $\Theta_{h}$.

The same observation actually holds for the MITC7 element, where the choice

$$
W_{h}=\left\{\zeta\left|\zeta \in H_{0}^{1}(\Omega), \zeta\right|_{T} \in S_{7}(T) \forall T\right\}
$$

was also allowed if we took $\mathrm{BDFM}_{2}$ instead of $\mathrm{RT}_{1}$ in (13).

On the other hand, for $k \geqslant 3$, on triangular elements with the choice of polynomials of order $k+1$ for velocities and $k$ for pressure as a Stokes element, the choice (iii) (i.e. use of $\mathrm{RT}_{k}$ ) for $\Gamma_{h}$ is to be recommended, giving $W_{h}$ a space of piecewise polynomials of degree $k+1$. Finally, always on triangles, the use of the $P_{2}-P_{0}$ element for Stokes would suggest a choice of type (i) (i.e. use of $\mathrm{BDM}_{1}$ ) for $\Gamma_{h}$ since it produces as $W_{h}$ a space of piecewise polynomials of degree two.

We end this section by describing the operators $R$ which are associated with the choices (i)-(v) for $\Gamma_{h}$. Since we believe it might be useful for constructing other elements, we shall present the case of a general degree $k$ (in (i) $(\mathrm{v})$ we had $k=3$ ).

$$
\mathrm{BDM}_{k}=\left\{\delta \mid \delta \in\left(P_{k}\right)^{2}\right\}
$$

For every $\delta$ smooth in $T, R \delta \in \mathrm{BDM}_{k}$ is characterized by

$$
\left.\begin{array}{c}
\int_{e}(\delta-R \delta) \cdot \tau p_{k}(s) \mathrm{d} s=0 \quad \forall e \quad \text { edge of } T \\
\forall p_{k} \text { polynomial of degree } \leqslant k \text { on } e \\
\int_{T}(\delta-R \delta) \cdot \operatorname{rot} p_{k-1} \mathrm{~d} x \mathrm{~d} y=0 \quad \forall p_{k-1} \in P_{k-1}(T) \\
\int_{T}^{(\delta-R \delta) \cdot \nabla\left(b_{3} p_{k-2}\right) \mathrm{d} x \mathrm{~d} y=0} \quad \forall p_{k-2} \in P_{k-2}(T)
\end{array}\right\}
$$


where $b_{3}$ is the cubic bubble function equal to $\lambda_{1} \lambda_{2} \lambda_{3}$.

$$
\begin{aligned}
& \operatorname{BDFM}_{k}=\left\{\delta\left|\delta \in\left(P_{k}\right)^{2}, \delta \cdot \tau\right|_{e} \in P_{k-1} \forall e\right\} \\
& \int_{e}(R \delta-\delta) \cdot \tau p_{k-1}(s) \mathrm{d} s=0 \quad \forall e, \forall p_{k-1} \in P_{k-1}(e) \\
& \int_{T}(R \delta-\delta) \cdot \operatorname{rot}\left(p_{k-1}\right) \mathrm{d} x \mathrm{~d} y=0 \quad \forall p_{k-1} \in P_{k-1}(T) \\
& \int_{T}(R \delta-\delta) \cdot \nabla\left(b_{3} p_{k-2}\right) \mathrm{d} x \mathrm{~d} y=0 \quad \forall p_{k-2} \in P_{k-2}(T) \\
& \mathrm{RT}_{k-1}=\left\{\delta \mid \delta \in\left(P_{k-1}\right)^{2} \oplus \underline{x}^{\perp} P_{k-1}\right\} \\
& \int_{e}(R \delta-\delta) \cdot \tau p_{k-1}(s) \mathrm{d} s=0 \quad \forall e, \forall p_{k-1} \in P_{k-1}(e) \\
& \left.\int_{T}(R \delta-\delta) \cdot p_{k-1} \mathrm{~d} x \mathrm{~d} y=0 \quad \forall p_{k-1} \in\left(P_{k-1}(T)\right)^{2}\right\}
\end{aligned}
$$

For a rectangular element $K$ we have instead

$$
\mathrm{BDM}_{k}(K)=\left\{\delta \mid \delta \in\left(P_{k}\right)^{2} \oplus \nabla\left(x y^{k+1}\right) \oplus \nabla\left(x^{k+1} y\right)\right\}
$$

and $R$ is defined by

$$
\left.\begin{array}{l}
\int_{K}(R \delta-\delta) \cdot \tau p_{k}(s) \mathrm{d} s=0 \quad \forall e, \forall p_{k} \in P_{k}(e) \\
\int_{K}(R \delta-\delta) \cdot p_{k-2} \mathrm{~d} x \mathrm{~d} y=0 \quad \forall p_{k-2} \in\left(P_{k-2}(K)\right)^{2}
\end{array}\right\}
$$

Finally we have

$$
\mathrm{BDFM}_{k}(K)=\left\{\delta \mid \delta_{1} \in P_{k} \backslash\left\{x^{k}\right\}, \delta_{2} \in P_{k} \backslash\left\{y^{k}\right\}\right\}
$$

with

$$
\left.\begin{array}{ll}
\int_{e}(R \delta-\delta) \cdot \tau p_{k-1}(s) \mathrm{d} s=0 & \forall e, \forall p_{k-1} \in P_{k-1}(e) \\
\int_{K}(R \delta-\delta) \cdot p_{k-2} \mathrm{~d} x \mathrm{~d} y=0 & \forall p_{k-2} \in\left(P_{k-2}(K)\right)^{2}
\end{array}\right\}
$$

\section{CONCLUSIONS}

We presented a general strategy for constructing new elements for Reissner Mindlin plate bending problems. The strategy consists essentially in finding the following items:

1. A velocity pressure pair of spaces $\left(\Theta_{h}, Q_{h}\right) \subseteq\left(H_{0}^{1}\right)^{2} \times L^{2}$ that is good for Stokes problems.

2. A pair of spaces $\left(\Gamma_{h}, Q_{h}\right) \subseteq H_{0}($ rot $) \times L^{2}$ for mixed approximations of linear elliptic problems, 
and an operator $R$ such that the following diagram commutes:

$$
\begin{aligned}
& \stackrel{\text { rot }}{\longrightarrow} L^{2}(\Omega) \\
& R \downarrow \quad \downarrow P_{h} \\
& \Gamma_{h} \stackrel{\text { rot }}{\longrightarrow} Q_{h} \\
& \text { ( } \mathscr{H} \text { defined in (64) and } P_{h}=L^{2} \text {-projection) }
\end{aligned}
$$

3. A finite element space $W_{h}$ such that

$$
\nabla W_{h}=\left\{\delta \mid \delta \in \Gamma_{h}, \operatorname{rot} \delta=0\right\}
$$

A simplified analysis (considering only the limit case $t=0$ ) shows that, if we use $\Theta_{h}$ for approximating rotations, $W_{h}$ for approximating the transverse displacement and the term $R\left(\nabla w_{h}-\theta_{h}\right)$ (instead of $\left.\nabla w_{h}-\theta_{h}\right)$ for the shear strains, we have an element that does not lock and that converges with optimal rate. However, we should note the following:

(a) Our analysis does not imply that the elements presented will be uniformly good for every $t>0$. We only analysed the limit case $t=0$, but we believe that this is already a very meaningful test.

(b) Our analysis also does not imply that the shear stresses will converge with optimal rate. Our analysis only covers the transverse displacement and rotations. Our guess is, however, that the shear stresses should behave as the pressure in Stokes problems, and hence converge with optimal rate.

(c) Other Reissner-Mindlin elements, based on a different conception and thus escaping our analysis, might also be good elements. However, if we use a reduction operator $R$ as in $P_{t h}$ (Section 2) with $R\left(\nabla w_{h}\right)=\nabla w_{h}$ for $w_{h} \in W_{h}$, then we believe that our analysis becomes almost necessary.

Finally, we note that we have not studied in this paper the actual numerical efficiency of the proposed elements, and thus their practical use. Such study and numerical investigations regarding the above observations will be the object of future communications. ${ }^{20}$

\section{REFERENCES}

1. E. Dvorkin and K. J. Bathe, 'A continuum mechanics based four-node shell element for general nonlinear analysis', $J$. Eng. Comp., 1, 77-88 (1984).

2. K. J. Bathe and E. Dvorkin, 'A four-node plate bending element based on Mindlin-Reissner plate theory and a mixed interpolation', Int. j. numer. methods eng., 21, 367-383 (1985).

3. K. J. Bathe and F. Brezzi, 'A simplified analysis of two-plate bending elements-The MITC4 and MITC9 elements', in G. N. Pande and J. Middleton (eds.), Numeta 87, Vol. I, Numerical Techniques for Engineering Analysis and Design, Martinus Nijhoff, Amsterdam, 1987.

4. K. J. Bathe and F. Brezzi, 'On the convergence of a four-node plate bending element based on Mindlin-Reissner plate theory and a mixed interpolation', in J. R. Whiteman (ed.), Proceedings of Conference on Mathematics of Finite Elements and Applications V, Academic Press, New York, 1985, pp. 491-503.

5. K. J. Bathe, Finite Element Procedures in Engineering Analysis, Prentice-Hall, Englewood Cliffs, N.J., 1982.

6. F. Brezzi and K. J. Bathe, 'Studies of finite element procedures-The inf-sup condition, equivalent forms and applications', in K. J. Bathe and D. R. J. Owen (eds.), Reliability of Methods for Engineering Analysis, Pineridge Press, Swansea, 1986.

7. F. Brezzi and M. Fortin, Mixed and Hybrid Finite Element Methods, to appear.

8. P. G. Ciarlet, The Finite Element Method For Elliptic Problems, North-Holland, Amsterdam, 1978.

9. P. A. Raviart and J. M. Thomas, 'A mixed finite element method for second-order elliptic problems', in Mathematical Aspects of Finite Element Methods, Lecture Notes in Mathematics, Vol. 606, Springer-Verlag, New York, 1975, pp. $292-315$ 
10. F. Breezi, J. Douglas, Jr., M. Fortin and L. D. Marini, 'Efficient rectangular mixed finite elements in two and three space variables', RAIRO $M_{2} A N, 21,581-604$ (1987).

11. K. J. Bathe and E. Dvorkin, 'A formulation of general shell elements-The use of mixed interpolation of tensorial components', Int. j. numer. methods eng., 22, 697-722 (1986).

12. M. Crouzeix and P. A. Raviart, 'Conforming and nonconforming finite element methods for solving the stationary Stokes equations', RAIRO Anal. Numer. R3, 33-76 (1973).

13. M. Fortin, 'Old and new finite elements for incompressible flows', Int. j. numer. methods fluids, 1, 347-364 (1981).

14. T. Sussman and K. J. Bathe, 'A finite element formulation for nonlinear incompressible elastic and inelastic analysis', $J$. Comp. Struct., 26, 357-409 (1987).

15. F. Brezzi and M. Fortin, 'Numerical approximation of Mindlin-Reissner plates', Math. Comp., 47, 151-158 (1986).

16. J. Douglas, Jr. and J. E. Roberts, 'Global estimates for mixed methods for second order elliptic equations', Math. Comp., 44, 39-52 (1985)

17. V. Girault and P. A. Raviart, Finite Element Approximation of the Navier-Stokes Equations, Lecture Notes in Mathematics, Vol. 749, Springer-Verlag, New York, 1979.

18. J. C. Nedelec, 'Mixed finite elements in $\mathbb{R}^{3}$ ' Numer. Math., 35, 315-341 (1980).

19. F. Brezzi, J. Douglas, Jr. and L. D. Marini, 'Two families of mixed finite elements for second order elliptic problems', Numer. Math., 47, 217-235 (1985).

20. K. J. Bathe, F. Brezzi and S. Cho, 'The MITC7 and MITC9 plate bending elements', Comp. Struct., in press. 